\title{
Optiques de Bragg-Fresnel pour la microfocalisation de rayons $\mathrm{X}$
}

\author{
G. Soullié, F. Legrand*, M. Idir**, A. Mirone**, P. Dhez*** et P. Chevallier**** \\ Laboratoire de Chimie-Physique, 11 rue P. \& M. Curie, 75231 Paris cedex 5, France \\ * CEA, Service Conception Expérimentale de la Mesure, BP. 12, 91680 Bruyères-le-Châtel, \\ France \\ ** LURE \& LSAI, Bât. 209D, Université Paris-Sud, 91405 Orsay cedex, France \\ *** LURE, Bât. 209D, Université Paris-Sud, 91405 Orsay cedex, France \& LPAN, Tour 12, \\ Université P. \& M. Curie, 4 place Jussieu, 75005 Paris, France
}

\begin{abstract}
RESUME
Nous présentons des résultats obtenus avec des optiques focalisant le rayonnement $X$. Ces optiques sont réalisées en gravant des réseaux à pas variable sur des multiconches par usinage ionique.

Ces optiques appelées lentilles de Bragg-Fresnel sont utilisées pour la microfluorescence $\mathrm{X}$ afin d'étudier la répartition d'éléments traces dans des petits échantillons.
\end{abstract}

\section{INTRODUCTION}

Depuis une dizaine d'années, les zones de Fresnel (ZF) en transmission sont utilisées pour leurs propriétés de focalisation [1]. Le principal domaine de longueurs d'onde d'application est celui de la fenêtre de l'eau $(4,4-2,3 \mathrm{~nm})$ dans lequel le contraste entre les molécules biologiques et l'eau est important.

Plus récemment sont apparues les zones de Bragg-Fresnel (ZBF) [2], Ces nouvelles optiques combinent les avantages de la bonne résolution spatiale des $\mathrm{ZF}$ avec la stabilité des réflecteurs de Bragg (cristaux ou multicouches) capables de résister aux flux $X$ intenses (synchrotrons, plasmas,...).

La résolution spatiale minimale est fixée par la taille du dernier créneau.

\section{LENTILLES LINEAIRES}

Ces lentilles peuvent être utilisées soit en diffraction classique, soit en diffraction conique [3].

Dans les deux cas, la ligne de focalisation monochromatisée est superposée à un fond de même longueur d'onde (réflexion spéculaire par la lentille); ce qui diminue le contraste. Pour remédier à cet effet, nous avons imaginé et réalisé un nouveau type de lentilles dites "hors-axe". On ne grave qu'une partie de la lentille sur le substrat multicouche de façon à séparer dans l'espace le faisceau focalisé du faisceau réfléchi spéculairement. En faisant varier l'angle d'incidence, on peut ainsi optimiser l'intensité de chaque ordre.

Nous avons développé une théorie dynamique pour les lentilles "centrées" et pour les lentilles "hors-axe". Dans ce calcul, la lentille est assimilée à une somme de réseaux périodiques[4]. 


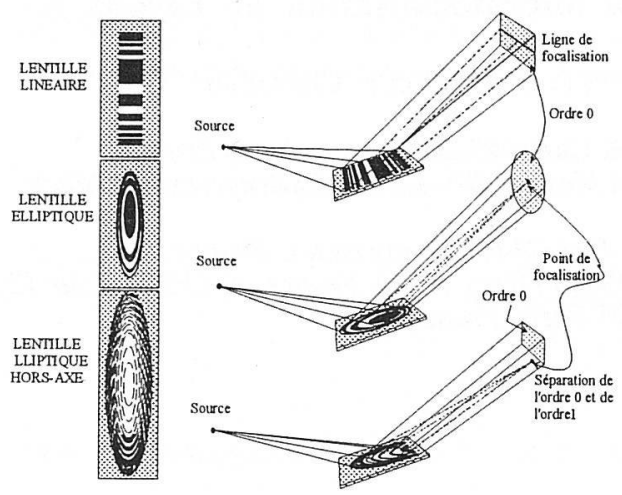

\section{LENTILLES ELLIPTIQUES}

Comme pour les lentilles linéaires centrées, le point focal est superposé à l'ordre zéro réfléchi par la lentille. Pour augmenter le contraste, on peut collimater le point focal. Il est également possible de concevoir et réaliser des lentilles elliptiques hors axe (figure 1)[5].

Fig. $l$ : Différentes lentilles de Bragg-Fresnel

\section{APPLICATION : MICROFLUORESCENCE X}

Un exemple d'application des lentilles de Bragg-Fresnel elliptiques est la microsonde par fluorescence X implantée sur l'anneau synchrotron DCI (LURE-Orsay). Une lentille elliptique gravée sur une multicouche $W / S i(d=3 \mathrm{~nm})$ focalise le rayonnement $X$ sur un échantillon fixé sur un support mobile. En déplaçant cet échantillon dans le plan focal, on établit la carte, point par point, de la répartition des éléments de l'échantillon.

Une expérience similaire a été effectuée sur une ligne onduleur de l'ESRF (Grenoble). Grâce à la qualité de la source (petite taille et brillance), un faisceau de $12 \mathrm{keV}$ a été focalisé dans un point de $2,5 \times 5 \mu \mathrm{m}^{2}$ (figure 2). Le flux mesuré par fluorescence était d'environ $10^{9} \mathrm{phot} / \mathrm{sec}$ (figure 3). Nous présenterons également une image d'un objet test en chrome de $800 \AA$ d'épaisseur, réalisée avec cette microsonde
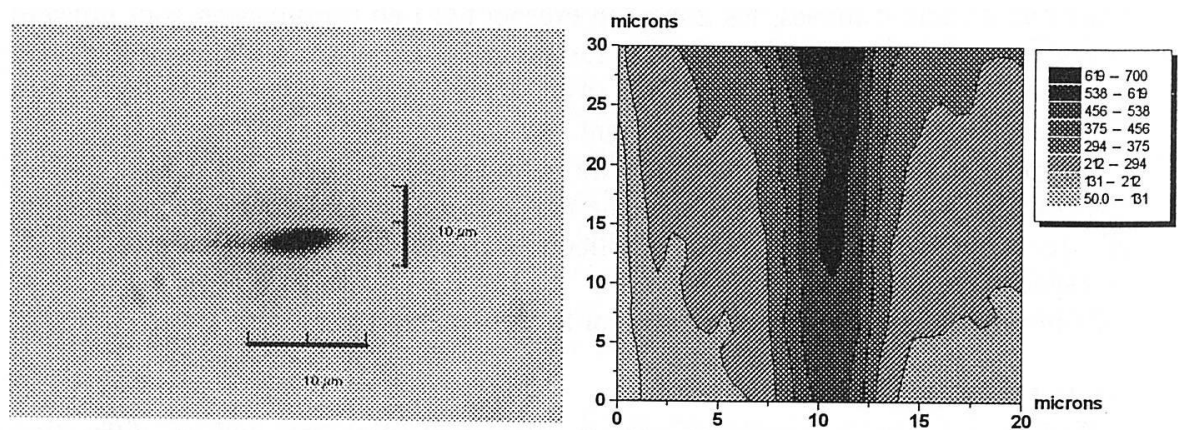

Fig. 2 : Photographie de la tache Fig. 3 : Image par fluorescence d'un objet test. focale.

(Intensité en coups/sec.)

\section{CONCLUSION}

Les premiers tests de lentilles elliptiques "hors-axe" ont déjà été réalisés, ils sont très encourageants. Nous envisageons de mettre en oeuvre une telle lentille pour la microsonde de fluorescence.

\footnotetext{
[1] G. Schmahl, X-rny microscopy :Nucl. Inst. Metl. 208 (1983) 361-365

[2] V.V. Aristov \& al., Revue Phys. Appl. 23 (1988) pp. 1623-1630

[3] M. Idir \& al., X-ray microscopy IV (1993), à paraître.

[4] A Mirone \& al., Opties Comm. (1994), à paraitre.

[5] A Mirone \& al. , X-ray microscopy IV (1993), à parâ̂tre.
} 